\title{
Ovarian metastasis from breast cancer mimicking a primary ovarian neoplasm: A case report
}

\author{
YOSHIKA AKIZAWA ${ }^{1}$, TOSHIYUKI KANNO ${ }^{1}$, YU HORIBE ${ }^{1}$, YUMI SHIMIZU ${ }^{2}$, \\ EIICHIRO NOGUCHI ${ }^{2}$, TOMOKO YAMAMOTO ${ }^{3,4}$, TAKAHIRO OKAMOTO ${ }^{2}$, \\ YOJI NAGASHIMA $^{3}$ and TSUTOMU TABATA ${ }^{1}$ \\ Departments of ${ }^{1}$ Obstetrics and Gynecology, ${ }^{2}$ Breast and Endocrine Surgery and \\ ${ }^{3}$ Surgical Pathology, ${ }^{4}$ Division of Pathological Neuroscience, Department of Pathology, \\ Tokyo Women's Medical University, Shinjuku, Tokyo 162-8666, Japan
}

Received August 19, 2020; Accepted April 23, 2021

DOI: $10.3892 / \mathrm{mco} .2021 .2297$

\begin{abstract}
Ovarian metastasis (OM) from breast cancer accounts for $3-38 \%$ of all ovarian neoplasms and is associated with various characteristic clinical presentations, such as pseudo-Meigs syndrome and Krukenberg tumor. Accurate diagnosis of OM may be challenging, as such lesions are frequently asymptomatic until they reach a large size. Occasionally, metastatic ovarian cancer is detected prior to the diagnosis of the primary tumor. Immunohistochemistry plays an important role in distinguishing primary ovarian tumors from extraovarian tumor metastases and may be helpful for identifying the primary tumor site. We herein report a case of OM from breast cancer masquerading as primary ovarian cancer. However, the correct diagnosis was made based on histopathological and immunohistochemical examinations. The patient had bilateral breast cancer, namely invasive lobular carcinoma of the left breast and ductal carcinoma of the right breast. Due to the presence of bilateral synchronous breast tumors, the possibility that the patient had hereditary breast and ovarian cancer syndrome or other relevant genetic factors was considered. Immunohistochemistry plays a key role in distinguishing between primary ovarian tumors and $\mathrm{OM}$, and it was also important for confirming the metastatic nature of the ovarian lesion and diagnosing the primary tumor in the present study.
\end{abstract}

\section{Introduction}

Ovarian cancer presents with a diverse range of findings. Ovarian metastasis (OM), in particular, is not uncommon.

Correspondence to: Dr Yoshika Akizawa, Department of Obstetrics and Gynecology, Tokyo Women's Medical University, 8-1 Kawada-cho, Shinjuku, Tokyo 162-8666, Japan

E-mail: akizawa.yoshika@twmu.ac.jp

Key words: metastatic ovarian cancer, invasive lobular carcinoma, simultaneous tumor, hereditary breast and ovarian cancer syndrome
Such tumors mainly originate from the colon, stomach, endometrium, appendix or breast $(1,2)$. Approximately $15 \%$ of ovarian tumors are metastatic (3). Bilateral small, solid and highly vascularized ovarian masses are suggestive of OM from breast cancer (4-8). Tumors may metastasize to the ovaries through various routes, including direct, hematogenous and lymphatic spread, as well as transcoelomic dissemination (9).

Breast cancer is the most common malignant tumor and one of the main causes of cancer-related mortality among women worldwide (10). Under $10 \%$ of patients with breast cancer exhibit evidence of distant metastasis at the time of initial diagnosis (11). OM from breast cancer is frequently asymptomatic until the tumor has grown to a considerable size. In cases of malignant tumors of unknown histology, immunohistochemical staining is performed as part of a thorough histological examination. First, the histological type of the tumor is grossly categorized as carcinoma, sarcoma, lymphoma, malignant melanoma or germ cell tumor. There are indicative immunohistochemical markers for each type, including cytokeratin $(\mathrm{CK})$ for carcinoma, vimentin and various differentiation markers for sarcoma, CD45 for lymphoma, S100 protein and melanosome-associated antigen (also known as clone HMB45) for malignant melanoma, and CD117/c-kit and Sal-like protein 4 for germ cell tumors. Once a tumor has been diagnosed as carcinoma, particularly metastatic carcinoma of unknown primary origin, immunohistochemistry is performed using a combination of antibodies against CK7, CK20 and tissue-specific antigens, such as thyroid transcription factor-1 (TTF1) for thyroid or lung cancer and caudal-type homeobox 2 (CDX2) for colorectal cancer. In the present case, these ancillary pathological procedures were also employed to reach a conclusive diagnosis (12). OM from breast cancer may occasionally mimic primary ovarian cancer. In recent years, our knowledge on hereditary breast cancer caused by genetic factors has increased. We herein report a case of OM from breast cancer mimicking primary ovarian cancer, in which the patient was diagnosed with synchronous bilateral breast cancer, indicating the possible involvement of genetic factors, such as breast cancer type 1 susceptibility protein $(B R C A) 1$ and BRCA2. 
A

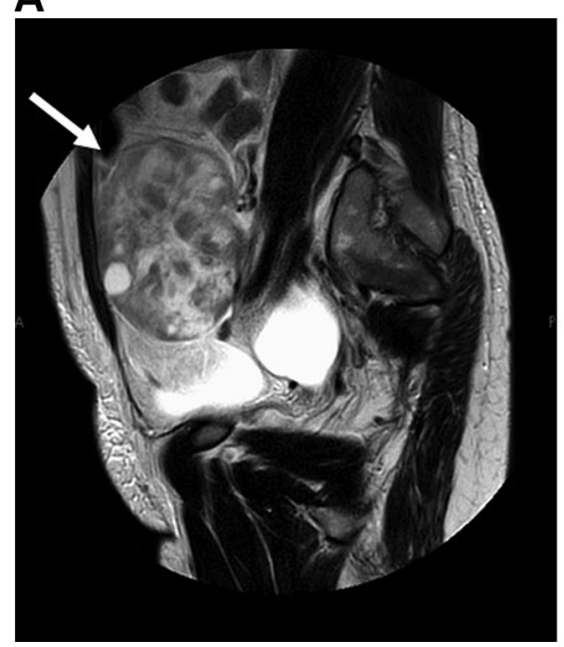

B

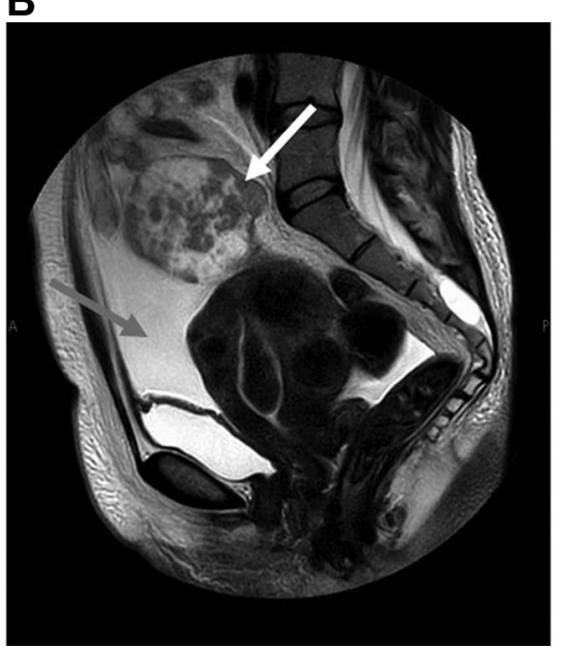

C

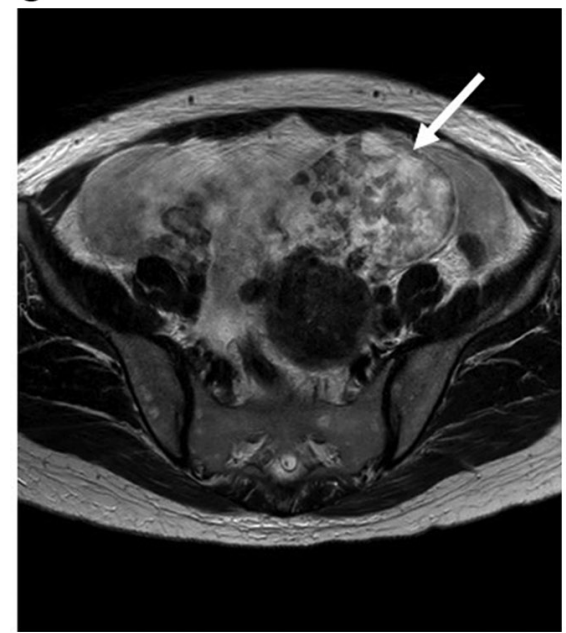

Figure 1. T2-weighted pelvic magnetic resonance imaging. (A and B) Sagittal and (C) axial images of the left ovarian carcinoma (white arrows) and massive ascites (gray arrows).

\section{Case report}

The patient was a 49-year-old Japanese woman, gravida 1, para 1 , who visited a local clinic complaining of abdominal distention lasting for 10 days. A left-sided ovarian tumor was identified on ultrasonography, and the patient was referred to Tokyo Women's Medical University Hospital for further examination and treatment in October 2019. Her medical history was unremarkable, and she had no family history of hereditary breast and ovarian cancer (HBOC). The patient had undergone menopause at the age of 48 years. Physical examination did not reveal any significant findings. Pelvic ultrasound examination revealed a large amount of ascitic fluid and a left-sided ovarian tumor. Pelvic magnetic resonance imaging examination revealed a left-sided ovarian tumor, measuring $11 \mathrm{~cm}$ in largest diameter, and a massive amount of ascitic fluid (Fig. 1). The patient's laboratory findings included elevated serum levels of cancer antigen (CA)125 (692 U/ml; normal range: $\leq 35 \mathrm{U} / \mathrm{ml})$ and CA15-3 (93.9 U/ml; normal range: $\leq 25 \mathrm{U} / \mathrm{ml})$. The patient was clinically diagnosed with ovarian carcinoma. Total abdominal hysterectomy, bilateral salpingo-oophorectomy and omentectomy were performed. Granulosa cell tumor, neuroendocrine carcinoma and metastatic ovarian cancer were considered in the differential diagnosis based on the morphological findings of the intraoperative frozen section biopsy. Further postoperative histopathological examination of the ovarian tumor raised the suspicion of metastasis from lobular carcinoma of the breast. A breast and endocrine surgeon at our hospital was consulted, and detailed examination revealed invasive lobular carcinoma (ILC) of the left breast (diameter: $5 \mathrm{~cm}$ ), invasive ductal carcinoma (IDC) in the right breast (diameter: $1 \mathrm{~cm}$ ) and suspected multiple metastases to the lumbar vertebrae. Genetic counseling and testing of $B R C A 1$ and $B R C A 2$ were performed, which did not reveal any germline mutations. The patient was started on 125-mg palbociclib tablets (once daily for 21 days followed by a break of 7 days), 2.5-mg letrozole tablets (once daily) and $120 \mathrm{mg}$ denosumab (once monthly). The patient has remained well, with stable disease. She has been to the hospital every 4 weeks for treatment and hematological examinations. Imaging examinations were also performed every 3-6 months, and the patient has remained stable (last follow-up, March 2021).

\section{Pathological findings of the ovarian tumor}

Intraoperative pathological findings. During the intraoperative pathological examination, the histological type of the tumor could not be determined. The tumor was composed of tightly packed, uniform, small round cells (Fig. 2) and was tentatively reported as a 'small round cell tumor'. Sex cord tumor, neuroendocrine carcinoma and metastatic carcinoma were considered as diagnostic candidates.

Gross findings. The left ovary was enlarged, solid, and measured 105x85x45 mm (Fig. 3). Its surface was smooth and tense. Its cut surface was lobulated, homogenously whitish in color, and firm in consistency. The right ovary was atrophic and small. The uterus and oviducts were unremarkable.

Histopathological finding s of the ovarian tumor. The resected specimens were immediately fixed with $10 \%$ formalin, embedded in paraffin, cut into $4-\mu \mathrm{m}$ sections and subjected to histopathological examination. On hematoxylin and eosin staining, the ovarian tumor was composed of small, uniformly round tumor cells without intercellular connections, with a high nuclear:cytoplasmic ratio. Occasionally, the nuclei of the tumor cells were eccentrically situated within intracytoplasmic mucus. Due to the possibility of the ovarian tumor being a metastatic carcinoma rather than a primary ovarian tumor, ancillary immunohistochemical studies were performed to identify the primary site. Among the potential primary sites, the stomach (poorly differentiated adenocarcinoma) and breast (ILC) were considered to be the most likely, based on the characteristics of the disease and the morphology of the tumor. The tumor cells were positive for CK (clones AE1/AE3 and CAM5.2) and CK7, but not CK20. Additional immunostaining revealed positivity 


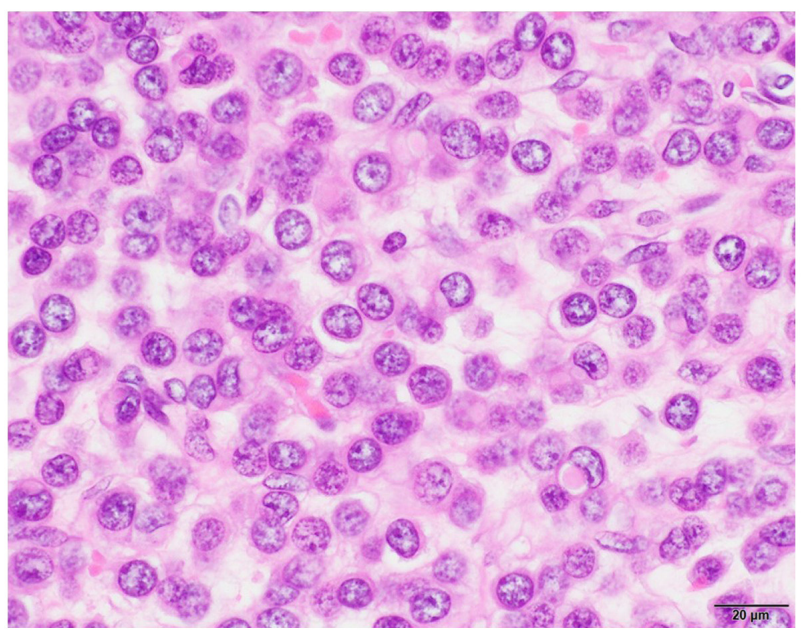

Figure 2. Histopathological examination of the left ovary revealed a small round-cell tumor. Hematoxylin and eosin staining. Scale bar: $20 \mu \mathrm{m}$; magnification, $\mathrm{x} 40$

for the estrogen receptor (ER) and negative results for TTF1 and CDX2. Accordingly, the breast was considered as the likely primary site. To evaluate the possibility of lobular carcinoma, E-cadherin expression was examined in the tumor, which produced a negative result (Fig. 4). Based on these findings, detailed breast examinations we performed, and bilateral breast carcinomas were detected. One was diagnosed as IDC and the other was diagnosed as ILC, the latter being the primary tumor of the OM.

Pathology of the breast tumors. Accordingly, detailed examinations and core-needle biopsies of the bilateral breast tumors were performed. The histological findings are shown in Fig. 5. Histologically, the left breast tumor was an ILC, which was composed of small tumor cells (Fig. 5A) and was immunohistochemically negative for E-cadherin (Fig. 5B). The ovarian tumor was composed of similar small uniform tumor cells (Fig. 5G), which were also negative for E-cadherin (Fig. 5H). By contrast, the right breast tumor was an IDC, exhibiting formation of neoplastic ducts (Fig. 5M) and positivity for E-cadherin (Fig. 5N). The molecular subtype of the breast ILC was classified as luminal A [positive for ER (Fig. 5C) and progesterone receptor (PgR; Fig. 5D), but negative for human epidermal growth factor receptor 2 (HER2; Fig. 5E)], which was the same staining pattern as that of the metastatic lesion (Fig. 5I, J and K, respectively). Ki-67-positive cells were scarce (Fig. 5L). The IDC in the right breast was basal-like [negative for the ER, PgR and HER2 (Fig. 5O, P and Q, respectively)], with a Ki-67 labeling index of $\sim 5 \%$ (Fig. 5R).

\section{Discussion}

The ovary is a common target of cancer metastasis, and OM exhibits characteristic clinical presentations, such as Krukenberg tumors (13) or pseudo-Meigs syndrome (14-16). However, it has been reported that the primary tumor could not be identified in $15 \%$ of OM cases (17). The incidence of $\mathrm{OM}$ in patients with breast cancer ranges between 13 and

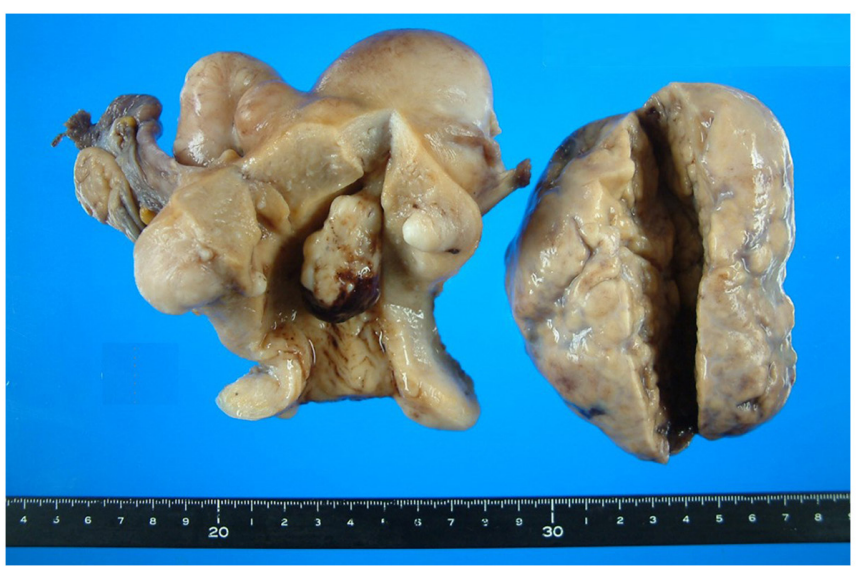

Figure 3. Macroscopic appearance of the surgical specimen. Left, uterus and right adnexa; right, left ovarian tumor.

$47 \%$ (these percentages are mainly based on autopsies or prophylactic or therapeutic oophorectomies) (18-20). OM from breast cancer is mainly found after surgery at the primary site. The mean duration of the period between the diagnosis of the primary tumor and the diagnosis of $\mathrm{OM}$ is $\sim 2$ years (21). OM from breast cancer is generally asymptomatic until the metastatic mass has reached a certain size, and such tumors frequently manifest as bilateral, solid, small ovarian masses. Some patients may present with other symptoms, such as ascites, gastrointestinal symptoms, pelvic pain and vaginal bleeding (22-24). Metastatic ovarian cancer can be diagnosed based on pathological examination, as was seen in the present case. In cases of breast cancer, both ILC and IDC have been reported to metastasize to the ovaries. In a study of 29 cases of OM from breast cancer, 12 of the 29 cases involved ILC (25). In another study, 2,605 cases of ILC or IDC of the breast were encountered during the 18-year study period. ILC and IDC accounted for $359(13.8 \%)$ and $2,246(86.2 \%)$ of the cases, respectively. Among those, there were $7(1.9 \%)$ cases of OM from ILC and $13(0.6 \%)$ cases of OM from IDC (26). In several studies, the pattern of metastasis from breast cancer was investigated, and ILC was found to affect the internal reproductive organs more often than IDC $(26,27)$. In the present case, the patient had simultaneous bilateral breast carcinomas, namely ILC in the left breast and IDC in the right breast, and the OM originated from the ILC in the left breast. Less than $10 \%$ of patients with breast cancer display evidence of distant metastasis at the initial diagnosis. On the other hand, patients with a history of breast cancer are 3-7 times more likely to develop primary ovarian cancer than OM $(8,28,29)$. As the present case involved simultaneous bilateral breast cancers, it is possible that the patient had HBOC syndrome. In cases of breast cancer involving mutations in the $B R C A$ genes, poly(ADP)-ribose polymerase (PARP) inhibitors are highly effective when used as adjuvant treatment $(30,31)$. $B R C A 1$ and $B R C A 2$ germline mutations are identified in $\sim 10 \%$ of all breast cancers, and PARP inhibitors and platinum-based chemotherapies are considered as suitable treatments for such cases (32). In addition, prophylactic risk-reducing surgery (risk-reducing mastectomy and/or 
A

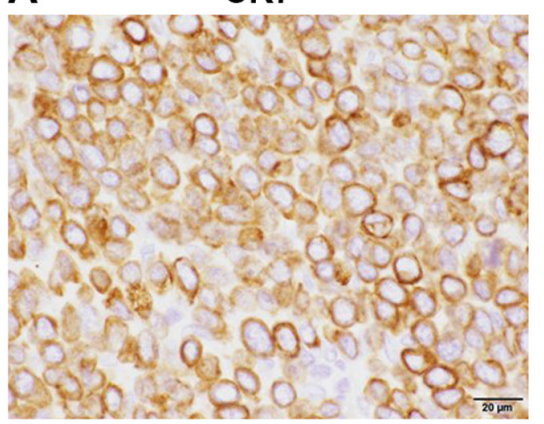

B

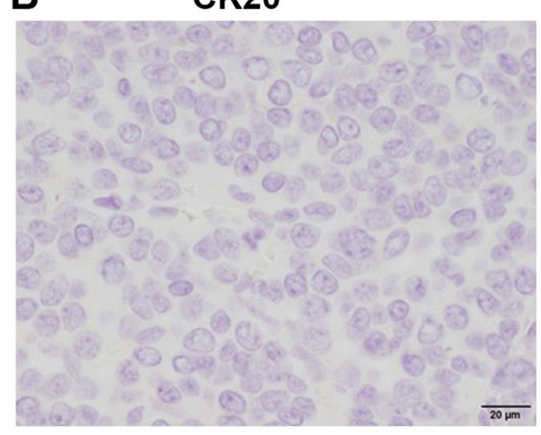

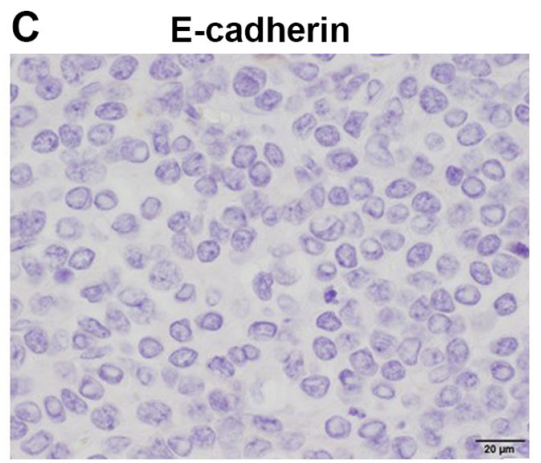

Figure 4. Immunohistochemical examination of the ovarian tumor. The tumor cells were positive for (A) CK7, and negative for (B) CK20 and (C) E-cadherin. Scale bar: $20 \mu \mathrm{m}$; magnification, $\mathrm{x} 40$. CK, cytokeratin.

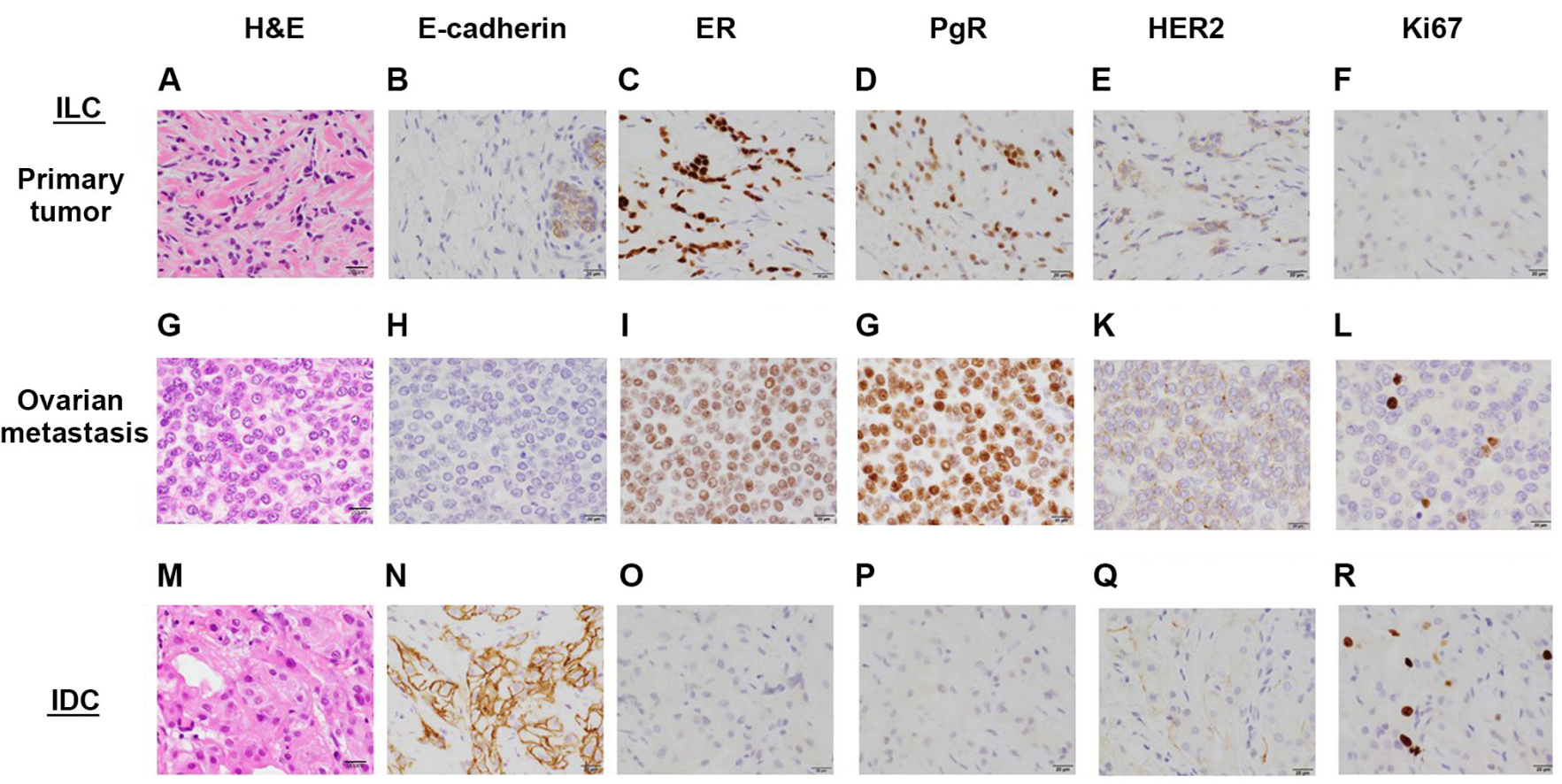

Figure 5. Pathological examination of the breast and ovarian cancers. The primary tumor was found to be ILC of the left breast based on (A) HE, (B) E-cadherin, (C) ER staining, (D) PgR, (E) HER2 and (F) Ki-67 staining. (G-L) Respective staining results for the ovarian metastasis. (M-R) Respective staining results for the IDC of the right breast. Scale bars: $20 \mu \mathrm{m}$; magnification, x40. ILC, invasive lobular carcinoma; IDC, invasive ductal carcinoma, HE, hematoxylin and eosin; ER, estrogen receptor; PgR, progesterone receptor; HER2, human epidermal growth factor receptor.

salpingo-oophorectomy) should be considered for patients that harbor $B R C A 1$ or $B R C A 2$ germline mutations. In cases in which two or more primary breast cancers develop, it is recommended that genetic testing of $B R C A 1 / 2$ should be carried out to identify any relevant genetic changes (33). Since our patient developed simultaneous bilateral breast cancers, it cannot be ruled out that other genetic factors may have been involved.

In conclusion, the differential diagnosis of OM and primary ovarian cancer based on clinical findings alone may be challenging, and clinicians should be aware that OM from breast cancer may occasionally masquerade as primary ovarian cancer.

\section{Acknowledgements}

Not applicable.

\section{Funding}

The present study was supported, in part, by the SHISEIKAI Scientific Award 2019 to YA.

\section{Availability of data and materials}

All data generated or analyzed during the present study are included in this published article.

\section{Authors' contributions}

YA, TY, YN, TO and TT collaborated in the conception and design of the study. TY and YN performed the pathological diagnosis of the ovarian and breast lesions. YA and YN wrote the manuscript. TK, YH, TY and YN were involved in figure preparation. TK, YH, YS, EN, YA and YN reviewed the 
manuscript. TK, YH, YS, EN, YA and YN analyzed the data and reviewed the manuscript. All the authors were involved in the preparation of the manuscript. All the authors have read and approved the final manuscript.

\section{Ethics approval and consent to participate}

Not applicable.

\section{Patient consent for publication}

Written informed consent for the publication of the clinical details of this case was obtained from the patient.

\section{Competing interests}

The authors declare that they have no competing interests.

\section{References}

1. Kubeček O, Laco J, Špaček J, Petera J, Kopecký J, Kubečková A and Filip S: The pathogenesis, diagnosis, and management of metastatic tumors to the ovary: A comprehensive review. Clin Exp Metastasis 34: 295-307, 2017.

2. Omranipour R and Abasahl A: Ovarian metastases in colorectal cancer. Int J Gynecol Cancer 19: 1524-1528, 2009.

3. Alvarado-Cabrero I, Rodríguez-Gómez A, Castelan-Pedraza J and Valencia-Cedillo R: Metastatic ovarian tumors: A clinicopathologic study of 150 cases. Anal Quant Cytopatho Histpathol 35: 241-248, 2013

4. Yadav BS, Sharma SC, Robin TP, Sams S, Elias AD, Kaklamani V, Kelly Marcom P, Schaefer S and Morris GJ: Synchronous primary carcinoma of breast and ovary versus ovarian metastases. Semin Oncol 42: e13-e24, 2015

5. Guerriero S, Alcazar JL, Pascual MA, Ajossa S, Olartecoechea B and Hereter L: Preoperative diagnosis of metastatic ovarian cancer is related to origin of primary tumor. Ultrasound Obstet Gynecol 39: 581-586, 2012.

6. Gagnon Y and Têtu B: Ovarian metastases of breast carcinoma A clinicopathologic study of 59 cases. Cancer 64: 892-898, 1989

7. Bigorie V, Morice P, Duvillard P, Antoine M, Cortez A, Flejou JF, Uzan S, Darai E and Barranger E: Ovarian metastases from breast cancer: Report of 29 cases. Cancer 116: 799-804, 2010.

8. Tserkezoglou A, Kontou S, Hadjieleftheriou G, Apostolikas N, Vassilomanolakis M, Sikiotis K, Salamalekis E, Tseke P and Magiakos G: Primary and metastatic ovarian cancer in patients with prior breast carcinoma. Pre-operative markers and treatment results. Anticancer Res 26: 2339-2344, 2006.

9. Yamanishi Y, Koshiyama M, Ohnaka M, Ueda M, Ukita S, Hishikawa K, Nagura M, Kim T, Hirose M, Ozasa H and Shirase T: Pathways of metastases from primary organs to the ovaries. Obstet Gynecol Int 2011: 612817, 2011.

10. Ghoncheh M, Pournamdar Z and Salehiniya H: Incidence and mortality and epidemiology of breast cancer in the world. Asian Pac J Cancer Prev 17: 43-46, 2016.

11. Ernst MF, van de Poll-Franse LV, Roukema JA, Coebergh JW, van Gestel CM, Vreugdenhil G, Louwman MJ and Voogd AC: Trends in the prognosis of patients with primary metastatic breast cancer diagnosed between 1975 and 2002. Breast 16:344-351,2007.

12. Stelow EB and Yaziji H: Immunohistochemistry, carcinomas of unknown primary, and incidence rates. Semin Diagn Pathol 35: 143-152, 2018.

13. Studzinski Z and Zajewski W: Bilateral metastatic ovarian tumors (Krukenberg's tumors) in the course of stomach cancer. Arch Gynecol Obstet 267: 95-97, 2002.

14. Kawakubo N, Okido M, Tanaka R, Mitsugi K, Fukuhara M, Aishima S, Kato $\mathrm{M}$ and Ichimiya $\mathrm{H}$ : Pseudo-Meigs' syndrome associated with breast cancer metastasis to both ovaries: Report of a case. Surg Today 40: 1148-1151, 2010.
15. Naito K, Oura S, Yasuoka $\mathrm{H}$ and Okamura Y: A case of pseudo-meigs' syndrome associated with ovarian metastases from breast cancer. J Breast Cancer 15: 474-477, 2012.

16. Fujii M, Okino M, Fujioka K, Yamashita K and Hamano K: Pseudo-Meigs' syndrome caused by breast cancer metastasis to both ovaries. Breast Cancer 13: 344-348, 2006.

17. Jung YE, Lee JW, Kim BG and Bae DS: Ovarian metastasis from pulmonary adenocarcinoma. Obstet Gynecol Sci 56: 341-344, 2013.

18. Rosendahl M, Timmermans Wielenga V, Nedergaard L, Kristensen SG, Ernst E, Rasmussen PE, Anderson M, Schmidt KT and Andersen CY: Cryopreservation of ovarian tissue for fertility preservation: No evidence of malignant cell contamination in ovarian tissue from patients with breast cancer. Fertil Steril 95: 2158-2161, 2011.

19. Peters IT, van Zwet EW, Smit VT, Liefers GJ, Kuppen PJ, Hilders CG and Trimbos JB: Prevalence and risk factors of ovarian metastases in breast cancer patients $<41$ years of age in the Netherlands: A nationwide retrospective cohort study. PLoS One 12: e0168277, 2017

20. Bastings L, Beerendonk CC, Westphal JR, Massuger LF, Kaal SE, van Leeuwen FE, Braat DD and Peek R: Autotransplantation of cryopreserved ovarian tissue in cancer survivors and the risk of reintroducing malignancy: A systematic review. Hum Reprod Update 19: 483-506, 2013.

21. Laifer S, Buscema J, Parmley TH and Rosenshein NB: Ovarian cancer metastatic to the breast. Gynecol Oncol 24: 97-102, 1986.

22. Ayhan A, Guvenal T, Salman MC, Ozyuncu O, Sakinci M and Basaran M: The role of cytoreductive surgery in nongenital cancers metastatic to the ovaries. Gynecol Oncol 98: 235-241, 2005.

23. Eitan R, Gemignani ML, Venkatraman ES, Barakat RR and Abu-Rustum NR: Breast cancer metastatic to abdomen and pelvis: Role of surgical resection. Gynecol Oncol 90: 397-401, 2003.

24. Moore RG, Chung M, Granai CO, Gajewski W and Steinhoff MM: Incidence of metastasis to the ovaries from nongenital tract primary tumors. Gynecol Oncol 93: 87-91, 2004.

25. Li CI, Anderson BO, Daling JR and Moe RE: Trends in incidence rates of invasive lobular and ductal breast carcinoma. JAMA 289: 1421-1424, 2003.

26. Borst MJ and Ingold JA: Metastatic patterns of invasive lobular versus invasive ductal carcinoma of the breast. Surgery 114: 637-641; discussion 641-632, 1993.

27. Lamovec $\mathrm{J}$ and Bracko M: Metastatic pattern of infiltrating lobular carcinoma of the breast: An autopsy study. J Surg Oncol 48: 28-33, 1991.

28. Curtin JP, Barakat RR and Hoskins WJ: Ovarian disease in women with breast cancer. Obstet Gynecol 84: 449-452, 1994.

29. Simpkins F, Zahurak M, Armstrong D, Grumbine F and Bristow R: Ovarian malignancy in breast cancer patients with an adnexal mass. Obstet Gynecol 105: 507-513, 2005.

30. Moore K, Colombo N, Scambia G, Kim BG, Oaknin A, Friedlander M, Lisyanskaya A, Floquet A, Leary A and Sonke GS, et al: Maintenance olaparib in patients with newly diagnosed advanced ovarian cancer. N Engl J Med 379: 2495-2505, 2018.

31. Robson M, Im SA, Senkus E, Xu B, Domchek SM, Masuda N, Delaloge S, Li W, Tung N, Armstrong A, et al: Olaparib for metastatic breast cancer in patients with a germline BRCA mutation. N Engl J Med 377: 523-533, 2017.

32. Economopoulou P, Dimitriadis G and Psyrri A: Beyond BRCA: New hereditary breast cancer susceptibility genes. Cancer Treat Rev 41: 1-8, 2015.

33. NCCN Clinical Practice Guidelines in Oncology Genetic/ Familial High-Risk Assessment; Breast, Ovarian, and Pancreatic. Version 2.2021-November 20,2020.

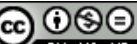

This work is licensed under a Creative Commons Attribution-NonCommercial-NoDerivatives 4.0 International (CC BY-NC-ND 4.0) License. 